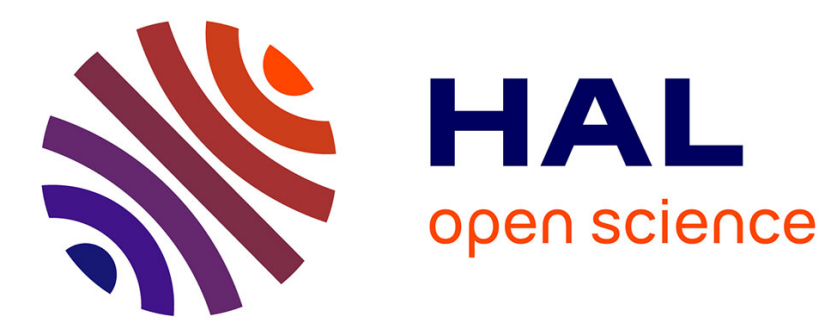

\title{
Distributed Efficient \& Fair Anticollision for RFID Protocol
}

\author{
Abdoul Aziz Mbacké, Nathalie Mitton, Hervé Rivano
}

\section{To cite this version:}

Abdoul Aziz Mbacké, Nathalie Mitton, Hervé Rivano. Distributed Efficient \& Fair Anticollision for RFID Protocol. WiMob 2016 - IEEE 12th International Conference on Wireless and Mobile Computing, Networking and Communications, Oct 2016, New York, United States. hal-01351350

\author{
HAL Id: hal-01351350 \\ https://hal.inria.fr/hal-01351350
}

Submitted on 21 Oct 2016

HAL is a multi-disciplinary open access archive for the deposit and dissemination of scientific research documents, whether they are published or not. The documents may come from teaching and research institutions in France or abroad, or from public or private research centers.
L'archive ouverte pluridisciplinaire HAL, est destinée au dépôt et à la diffusion de documents scientifiques de niveau recherche, publiés ou non, émanant des établissements d'enseignement et de recherche français ou étrangers, des laboratoires publics ou privés. 


\section{Distributed Efficient \& Fair Anticollision for RFID protocol}

\author{
Abdoul Aziz Mbacke \\ Inria, France \\ aziz.mbacke@inria.fr
}

\author{
Nathalie Mitton \\ Inria, France \\ nathalie.mitton@inria.fr
}

\author{
Herve Rivano \\ Univ Lyon, INSA Lyon, CITI, France \\ herve.rivano@inria.fr
}

\begin{abstract}
RFID technology suffers from a recurring issue: the reader-to-reader collision. Numerous protocols have been proposed to attempt to reduce them, but, remaining reading errors still heavily impact the performances and fairness of dense RFID deployments. This paper introduces a new Distributed Efficient \& Fair Anticollision for RFID (DEFAR) protocol. It reduces both monochannel and multichannel collisions as well as interference by a factor of almost $90 \%$ in comparison with the best state of the art protocols. The fairness of the medium access among the readers is improved to a $99 \%$ level. Such improvements are achieved applying a TDMA-based "serverless" approach and assigning different priorities to readers depending on their behavior over precedent rounds. A distributed reservation phase is organized between readers with at least one winning reader afterwards. Then, multiple reading phases occur within a single frame in order to obtain fast coverage and high throughput. The use of different reader priorities based on reading behaviors of previous frames also contributes to improve both fairness and efficiency. Simulation results show the robustness of the proposed solution in terms of different metrics such collision avoidance, fairness and coverage and in comparison with a centralized literature solution.
\end{abstract}

Keywords-RFID Radio Frequency Identification ; reader anticollision problem ; MAC layer ; resource allocation ; distributed systems ; mobile systems

\section{INTRODUCTION}

RFID is a technology that allows the contactless and out of sight identification of different goods or individuals through the use of readers and tags associated with the goods or individuals in question. The principle is to emit a radio frequency signal towards tags which in return use the energy issued from the previously received signal to reflect their response [1]. This technique is known as back-scattering. RFID technology is nowadays met in numerous applications such as transportation systems, logistics, access control, security, etc. In some installations, one single reader is not enough to cover a specific identification area or the application might require the existence of more than one checking area. For instance, in the case of a warehouse where every good has a tag attached to it, it should be possible to track the different positions or movement of the goods inside the area (entrance/exit of goods, checkpoints, load/unload platforms, ...). In order to overcome the full coverage of the deployment area and propagation dispersion and scattering, dense RFID

This work was partially supported by a grant from CPER/FEDER DATA and IPL CityLab@Inria. systems are deployed. In the same instance of the warehouse, a different solution could be envisaged. Instead of relying on static RFID readers deployed on different gates inside the area, these warehouses could rely on human checkers equipped with hand held RFID readers. Equipping these persons with such devices that can be carried around the different parts of the warehouse can facilitate the task. However, such a promiscuity between RFID readers is responsible for recurring collision issues [2], [3], which in turn highly impacts the reading performances. As such, the design of an efficient reader anticollision protocol has emerged as one of the most interesting research issues in recent years. The main objective when building an efficient RFID system is to schedule readers' activities to reduce as much as possible interference and collisions in order to improve on throughput, delay and energy consumption. Also, the algorithm design should anticipate a potential deployment of mobile readers.

The literature review broadly shows two main approaches: TDMA-based relying on the use of a central server to add the needed layer of synchronization among the different readers [4], [5], [6] and CSMA-based relying on a monitoring of the radio channel and backoff schemes for a given period of time to ensure the medium is unoccupied [7], [8].

In this paper, we introduce DEFAR, a new anticollision algorithm for RFID. It is, to the best of our knowledge, the first distributed multichannel TDMA anticollision mechanism for RFID. Its main characteristics are as follows:

-distributed, local and scalable : each reader runs the same algorithm and relies only on local information sent by its neighbors. Unlike other TDMA-based approaches, DEFAR does not rely on the use of a central entity for synchronization but rather on a loose TDMA approach based on the readers internal clocks and time margins;

-multichannel: the four different frequencies standardized by ETSI [9] are leveraged in order to reduce collisions;

-fair: DEFAR provides the reader with a fair and balanced access to tags, reaching a Jain index of close to $100 \%$;

-efficient: DEFAR reduces the number of collisions when compared to the state of the art approaches by a factor of close to $90 \%$, reaching an efficiency level close to $100 \%$; -mobile-ready: the localized approach of the algorithm allows it to handle dynamic scenarios without impacting the different metrics explored. 
As every distributed anticollision protocol from the literature, DEFAR assumes 2 main communication channels, one between tags \& readers and one between readers. It uses a control channel for the purpose of beacons exchange between readers as well as the four available reading channels for communicating with the tags, making the readers selfaware of their surrounding neighbors as well as their reading behavior. It relies on the use of a beaconing mechanism organized in frames, themselves subdivided in slots. To allow each reader to access the tags in a fair manner, DEFAR introduces a priority scheduling, ensuring no reader spends more than 2 following frames without accessing the medium. Each time a reader accesses the medium, it is allocated a time slot long enough to retrieve the information of all tags laying in its reading range. Through extensive testing, we highlight the following engineering insight, DEFAR is a fair protocol with an equitable access to the medium. Latency is also reduced and maximum coverage of all tags in reading range quickly reached within the first 10 frames.

The rest of this paper is organized as follows, Section II reviews the problem statement and highlights our motivation before Section III explores some state-of-the-art anticollision protocols for RFID. In Section IV we detail the DEFAR algorithm, its performances are evaluated and confronted to the literature protocols in Section V. Section VI prospects the propagation range model and the concept of mobility applied to our proposal. Finally, Section VII concludes by discussing future research directions.

\section{Problem Statement}

\section{A. Collision issues}

Different types of collisions can be observed. When one reader accesses multiple tags at the same time, all interrogated tags backscatter their ID simultaneously resulting in a collision and tags failing to be identified. This kind of collisions is referred to as "tag collisions" in the literature and have been studied and solved with ALOHA [10], tree [11], and frame-and-tree [12] based protocols. This type of collisions is out of the scope of this paper. Another instance of collisions happens when multiple readers access a given tag concurrently. This type of collision is referred to as "reader-to-reader collision". Indeed, aiming at increasing the coverage area and/or improving the total reading delay in RFID systems, one solution was to increase the number of deployed readers. This reader densification unfortunately results in the growth of the number of collisions. In a single channel environment, collisions occur in the cases depicted in Figs. 1a and 1b. In Fig. 1a, multiple readers attempt to access a single tag simultaneously. Their reading requests collide on the common channel and the tag cannot be read. In order to avoid this kind of interference, readers should either operate at different times or operate at a distance of at least $d=2 \times d_{C R T}$ with $\mathrm{d}_{C R T}$ being the reading range of a reader. In the example of Fig. 1a, only Tags T1 and
T3 will be read resp. by readers R1 and R2. Another type of collision is depicted in Fig. 1b: this collision happens when the back-scattered response from a tag collides with the signal of a second neighboring reader. To overcome this problem, readers should either operate at different times or use different frequencies. In Fig. 1b, tags T1 and T3 will successfully be read by readers $\mathrm{R} 1$ and $\mathrm{R} 2$, while reading of T2 will suffer from the interference of R2.

Following the ETSI EN 302 208-2 regulation [9], solutions have been implemented using multichannel property. However, the consideration of a multichannel environment brought to light new issues regarding the interference that might be caused by adjacent channels in the same vicinity. As such, HAMAC (High Adaptive MAC Protocol)[8] addressed the adjacent channel interference issue and the adjacent channel range, $d_{A C}$, was introduced. If the distance between neighboring readers is greater than $d_{A C}=$ $3.3 \times d_{C R T}$, these interference are avoided. As illustrated in Fig. 1c, if readers R1 and R2 operate on different channels, tag T2 will still not be read since it still lies within the adjacent channel interference range of $\mathrm{R} 1$ and $\mathrm{R} 2$. T1 and T3 will not suffer from the interference range and will respectively be read by $\mathrm{R} 1$ and $\mathrm{R} 2$. In order to get a reading from all the tags, a configuration as the one shown in Fig.1d should be considered.

\section{B. Centralized vs Distributed}

In order to avoid collisions, a coordination among readers should be established. Several solutions have been introduced depending on how readers communicate. Overall, they follow one of the two following paradigms:

-centralized: the readers communicate with a superior entity (central server) which organizes reading rounds. These solutions are mainly adopted in TDMA-based approaches. They hardly support dynamics and mobility;

-distributed: the readers are able to communicate between each other directly in a peer-to-peer fashion and agree on their reading behaviors to avoid collisions.

While, at first glance, the first kind seems to be more suitable for readers collision issue, connecting every reader in a dense environment to a central server highly impacts the cost of deployment and induces delays that prevent from mobility handling, unlike distributed solutions.

\section{Monochannel vs Multichannel}

In the first versions of RFID systems, all readers had to communicate with tags using a common channel. This single frequency medium quickly resulted in excessive collisions. Multichannel has thus been introduced in the update of the standard [9]. Efficiently assigning different channels to neighboring readers reduces the number of collisions.

DEFAR makes good use of this enhancement by organizing the readings in rounds themselves divided in max_slots number of slots, by still taking care of interference that 




(a) Reading interference



(b) Interference range



(c) Adjacent channel collision

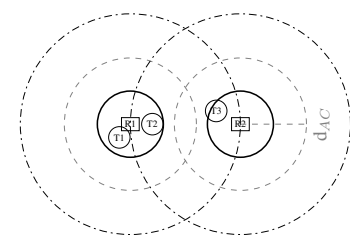

(d) Adjacent channel collision

Figure 1: Collisions

may occur even between different frequencies. This allows DEFAR to offer up to max_slots $\times 4$ neighboring readers to read tags during a frame.

\section{RELATED WORK}

This section browses different approaches, although not being exhaustive. We focus on the most significant works for DEFAR, ie five TDMA-based ones: DCS and VDCS also known as Colorwave, GDRA, an evolution of [5] which implements multichannel, and ACoRAS that inspired the idea in this algorithm for a better efficiency and fairness. We also present CSMA-based algorithms such as Pulse and a more recent algorithm, HAMAC, a multichannel approach that relies on the variation of the contention window.

[13] presented a monochannel distributed algorithm designed with a unique frame size for all readers. Every reader reserves a color (slot) within the frame to access tags and broadcasts the information to its neighbors. In case 2 or more readers collide e.g with the same color, they randomly chose new colors within the same frame size. The issue with such a system is that in dense deployments, the frame size may not be sufficient to contain all readers, resulting in consecutive collisions and throughput drops. Later [14] partially solves this issue by introducing thresholds on the number of collisions to allow repetitive colliding readers to expand their frame size as to have more room for available colors. However, having different frame sizes among asynchronous readers can turn out that color reservation may not be shared properly among readers, resulting in multiple collisions and throughput drop. Also having different frame sizes across the system induces a drop in fairness.

Later GDRA [4], to the best of our knowledge, the only TDMA-based multichannel algorithm of the literature, with a centralized approach, a central server sends on a dedicated channel (or medium) an Arrangement Command containing the number of available slots. Upon reception, every reader randomly chooses a channel among the four available [9] frequencies and a slot within the frame size using the Sift distribution [15]. Once at the corresponding slot, readers send a beacon to advertise their channel access. In case of multiple readers with the same channel and slot, all contending readers get disabled for the current frame. This algorithm considers the use of bi-static antennas to allow readers to receive other beacons while they are sending their own and also requires a dedicated control channel or medium with a server capable to reach all readers periodically. However, disabling all contending readers in case of a collision induces a throughput drop and efficiency loss. Furthermore, the contention technique used affects the fairness of the system since readers stuck in a denser area can be repeatedly colliding and being disabled while others are accessing the medium. Also, unlike DEFAR, GDRA does not take care of collisions that still exist even with different frequencies.

Hamouda \& al. [6] proposed ACoRAS, a TDMA-based single channel centralized algorithm in which readers are assigned colors (slots) by a central server in order to access tags in a first phase. The colors assignment is done following the construction of a Minimum Independent Set. This is done to make sure no two, or more, neighboring readers are assigned the same colors. During a second phase, the number of colors assigned to each reader is reduced according to the number of tags they cover to reduce latency. ACoRAS was an inspiration to our work to ensure no reader spends a defined period of time without accessing the medium. This characteristic taken into account allowed to build a fairer protocol, but the idea of designing a more dynamic protocol prevented us from using a central server for such purposes.

Regarding CSMA-based proposals, several algorithms were suggested. Birari \& Iyer proposed Pulse[7], a single channel CSMA-based distributed algorithm. It uses both a control and a data channel for collision avoidance. So while a reader accesses tags on the data channel, it periodically sends beacons to neighboring readers on a dedicated control channel. Such a solution comes at a high cost in terms of energy consumption regarding the number of beacons sent. Also the channel access backoff is not considered, so in case of simultaneous access from multiple readers, collisions may not be detected resulting in a lower throughput. Whereas in our proposal, a channel access mechanism based on a backoff before beaconing is organized.

More recently Amadou \& al. designed HAMAC[8], a CSMA-based, distributed multichannel algorithm. To the best of our knowledge, HAMAC is the only literature approach that manages collisions on tags between different frequencies. It works by defining an initial maximum value for the backoff contention window. This value is 
then decreased during backoff by each reader depending on the channel state. Although, such a solution may reduce collisions, having different contention frame sizes among readers can result in a drop of fairness among readers as well as an increase of latency for certain readers.

From this observation, we deduce that while centralized solutions seem to be the most performing ones, they are either not scalable for more dynamic deployments [6] or make significant trade-offs regarding throughput and efficiency for the sake of reducing collisions [4]. CSMA-based approach on the other hand by leveraging on contention window size among readers impact the fairness of access between readers and affects their energy consumption with readers having to either continuously emit a jamming signal or listen to the medium before reading tags [7], [8]. As such, we propose to focus on a distributed TDMA-based solution that could be better suit dynamic environments without the need of a central server and be able to offer a fair access to the medium to all readers while also being able to reduce collisions and improve throughput so as to be the most efficient possible.

\section{DESIGN OF DEFAR}

In regards of the drawbacks expressed in Section III, we designed the Distributed Efficient \& Fair Anticollision for RFID algorithm to ensure that at least one reader among the colliding ones would access the medium and read the tags in its vicinity. One of the main motivations when designing DEFAR was to support the deployment of dynamic RFID systems with static/mobile readers and/or tags. We also aimed at creating a distributed algorithm to avoid being dependent to a central server which would preclude our solution from being used in mobile applications and could also make the solution much more expensive to deploy regarding the communication between the readers and the central server. Unlike [4], our algorithm does not depend on the use of additional hardware. Another issue met in regular algorithms is to ensure that readers deployed in a given area get access to the medium in a fair and balanced manner. We attempt to resolve this issue by allocating different priority levels to readers to access tags.

\section{A. Overview of DEFAR}

DEFAR is a distributed multichannel TDMA RFID anticollision protocol. In order for readers to exchange beacons, a dedicated communication channel is used, different from the ones used to access tags. Two ranges are then identified: -the reading range $\mathrm{d}_{C R T}$, previously described in Section II, which is the range within which any laying tag will be successfully interrogated;

-the communication range $\mathrm{d}_{C o m}$ of readers, defined as $d_{C o m}=2 \times d_{A C}$, with $d_{A C}=3.3 d_{C R T}$ being the adjacent channel interference range, in order to overcome both the single channel and adjacent channel collisions presented in Section II. Setting this value for $d_{C o m}$ ensures no adjacent channel collision is conceivable since readers closer than $d_{A C}$ (as depicted in Fig 1d) will be able to exchange beacons and organize their readings. Thus, a reader which does not receive any beacon from the area defined by $d_{C o m}$ is sure not to collide with any other reader.

The medium access is divided into different time frames which are themselves again divided into max_slots. Each slot can be accessed by up to 4 readers in the same vicinity using one of the 4 channels [9]. This permits to have up to $N=$ max_slots $\times 4$ contending readers (as explained in Section II-C) in a defined vicinity against just $N^{\prime}=$ max_slots in a single channel environment. This reduces collisions by a 4 factor while improving the throughput by the same factor. The slots are organized into two different phases :

-First, a beaconing phase for each reader to discover its neighbors and ensure it can access the medium. During this phase each reader observes a random backoff period before sending its beacon. The way we designed the number of backoff slots allows an insubstantial number of eventual beacon collisions. A large range of beaconing slots are available (see Fig 2) for readers to randomly chose from and only readers at the current ongoing reading slot are awaken thus making beacon collisions very unlikely to happen.

-Second is a reading phase for readers to access tags for a defined period of time. Regarding the previous beacons exchange between readers and the disabling of colliding readers, no collision is then conceivable when reading tags, since on each slot and frequency, there cant be more than one reader trying to read tags : the corresponding competition is based on the information gathered during the beaconing phase and solved as follows.

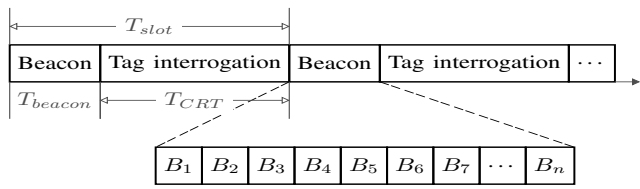

Figure 2: Frame design

The readers are able to follow the flow of frames with beaconing and reading phases thanks to a loose TDMA approach based on internal clocks and time margins. To overcome the general disabling of all readers in case of a collision, we introduce an ID comparison to allow at least one of the contending readers to access the tags. Also, in order to avoid the same readers to constantly access the tags, we create different priority levels to increase the chances of previously failing readers, while decreasing the ones for previously winning ones. We define 3 different priority levels for the readers. NEUTRAL is the priority of all readers at start. $L A Z Y$ is the lowest priority it is given to readers which have successfully read the tags in their range on the previous frame. PUMPED UP, the highest priority, is given to readers that have failed accessing the medium, thus giving them a 
higher probability of accessing tags during the following frame (see Fig 3).

At the system start, all the readers are in their NEUTRAL priority and wait for their chosen slot to come up. Each slot has a duration of $T_{\text {slot }}$. This duration is split into two parts, $T_{\text {beacon }}$ [9] used by readers to send beacons at the start of their slot and $T_{C R T}$ [5], [4], [8], [16] used to access and read tags. A frame can then be defined as $T_{\text {frame }}=$ max_slots $* T_{\text {slot }}$ with $T_{\text {slot }}=T_{\text {beacon }}+T_{C R T}$.

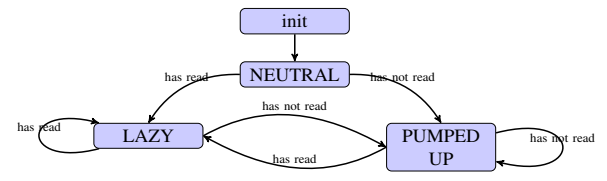

Figure 3: Priority levels diagram

\section{B. DEFAR}

At the beginning of the first frame, all readers randomly select a slot between $[0 ;$ max_slots [ and a channel among the available ones standardized by [9]. Knowing the length of a slot (as described in the previous subsection) readers then wait for their corresponding slot for a duration of $t=$ chosen_slot $* T_{\text {slot }}$ with the default NEUTRAL priority. Once at the corresponding chosen slot, a reader $\mathrm{R}_{i}$ observes a random backoff period before sending its beacon containing its ID and token on the dedicated communication channel. Two scenarii can occur (Algo. 1).

Scenario 1: $\mathrm{R}_{i}$ does not receive any beacon during $\mathrm{T}_{\text {beacon }}$. $\mathrm{R}_{i}$ is thus the only reader with this corresponding token in its vicinity. It can then access the medium to read tags during $\mathrm{T}_{C R T}$ and switches its priority to $L A Z Y$ (1. 5-6).

Scenario 2: $\mathrm{R}_{i}$ receives one or more beacon(s) with corresponding tokens during $\mathrm{T}_{\text {beacon }} . \mathrm{R}_{i}$ thus compares the IDs contained in the received beacons with its own:

- Either ID of $\mathrm{R}_{i}$ is the smallest. $\mathrm{R}_{i}$ then accesses to the medium for $\mathrm{T}_{C R T}$ and switches to $L A Z Y$ priority (1. 8-9);

- or ID of $\mathrm{R}_{i}$ is greater than any of the IDs received. $\mathrm{R}_{i}$ leaves the contention and switches its priority to PUMPED $U P$ (1. 10). It loses the previously chosen token and randomly picks another set of channel and slot for the next frame.

This process is kept throughout the entire first frame.

During the following frames, we keep the same collision resolving idea with a twist accounting the previously introduced priorities of the readers. Readers once again randomly select a slot and channel among the available ranges. Once the corresponding slot to the chosen token is up, reader $\mathrm{R}_{i}$ observes a random backoff period and sends its beacon with not only its ID and token but also its priority level. The following can happen (Algo. 2):

Scenario 1: $\mathrm{R}_{i}$ is $L A Z Y$ :

- no collision: there are no concurring readers in the vicinity of $\mathrm{R}_{i}$, it sends its beacon but does not receive any beacon from its neighbors. It then accesses the tags for $\mathrm{T}_{C R T}$ and remains in $L A Z Y$ priority (1. 5-6);

- collision: there is at least one concurring reader in the vicinity of reader $\mathrm{R}_{i}$ which receives at least one corresponding beacon from one of its neighbors (1. 7):

* $\mathrm{R}_{i}$ matches the priority levels of the readers that sent their beacons, if any of them is a PUMPED UP one, $\mathrm{R}_{i}$ shuts off and waits for the the next frame with a PUMPED UP priority (1. 9-10);

* if all the readers that have sent beacons are $L A Z Y$ as well, $\mathrm{R}_{i}$ then matches its ID with the readers that sent their beacons. It resolves the contention with respect to the IDs as done in the first round (1. 12-14).

Scenario 2: $\mathrm{R}_{i}$ is PUMPED UP: - no collision: there are no concurring readers in the vicinity of $\mathrm{R}_{i}$, it sends its beacon but does not receive any beacon from its neighboring readers. It then accesses the tags for $\mathrm{T}_{C R T}$ and switches its priority to $L A Z Y$ (1. 5-7);

- collision: there is at least one concurring reader in the vicinity of reader $\mathrm{R}_{i}$ which receives at least one corresponding beacon from one of its neighbors (1. 7):

* $\mathrm{R}_{i}$ compares the priority levels of the readers that have sent their beacons during phase 1 , if all of them are $L A Z Y$ ones, $\mathrm{R}_{i}$ accesses the medium to read tags for $\mathrm{T}_{C R T}$ and then switches to $L A Z Y$ priority (1. 18-19);

* if any of the readers that have sent a beacon is in a PUMPED UP priority as well, $\mathrm{R}_{i}$ then compares the IDs and resolves the contention with respect to the IDs as done in the first round (1. 21-23).



\section{Performance Evaluation}

In order to highlight the benefit brought by our algorithm, we implemented both DEFAR and GDRA using WSNet $^{1}$ to fairly evaluate their performances in various scenarii. WSNet is an event-driven simulator for large scale wireless networks. We considered a dense RFID system where 100

\footnotetext{
${ }^{1}$ http://wsnet.gforge.inria.fr/
} 


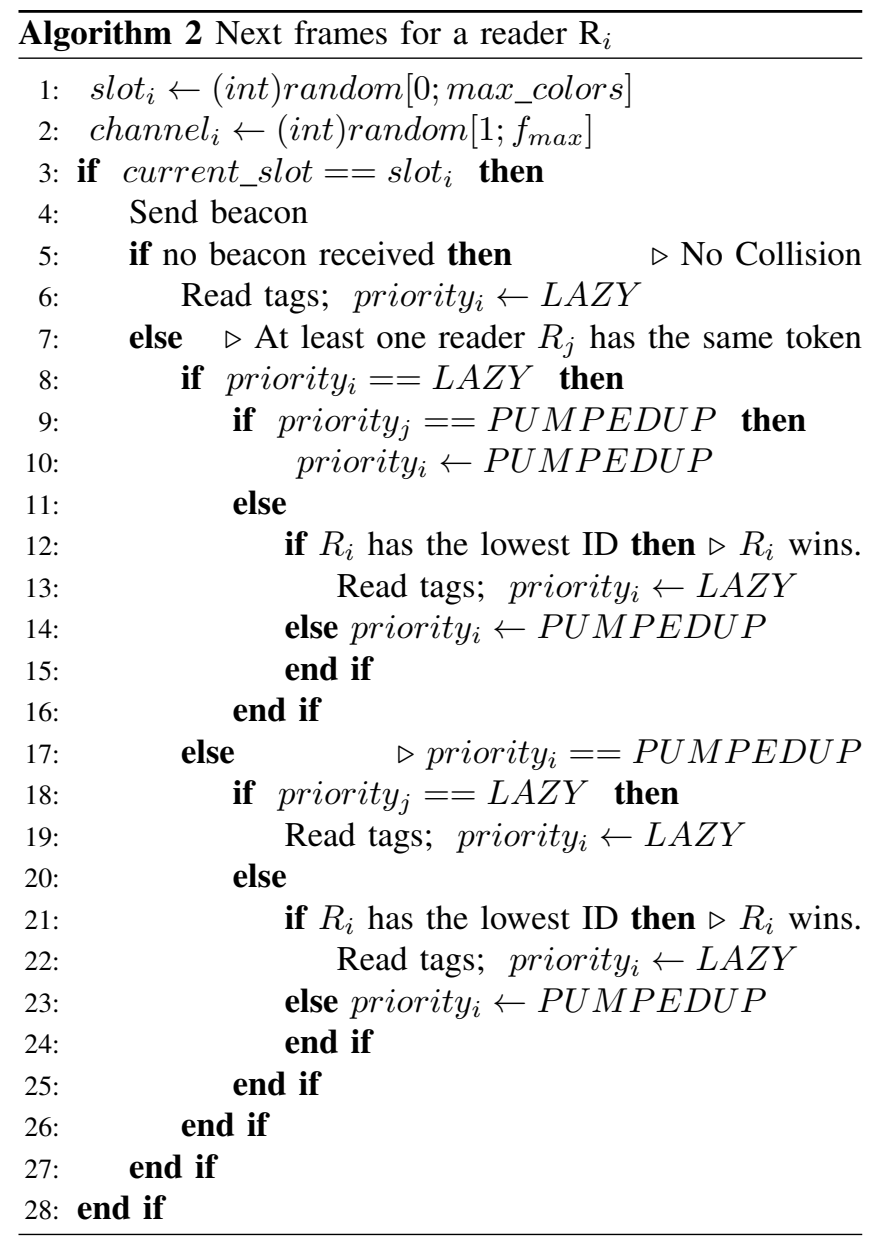

to 500 readers are randomly deployed uniformly on a 1000 $\times 1000$ meters square area with 2000 tags arranged. With such a deployment, we have a density of at least 1 colliding reader for each reader and a coverage of at least 3 tags with 100 readers deployed. With 500 readers deployed, each has at least 3 other concurring readers and a coverage of 4 tags. According to the reader maximum transmission power defined in [9], we set the reader to tag communication range to $d_{C R T}=10 \mathrm{~m}$ while the interference range is set to $d_{A C}=3.3 \times d_{C R T}$ and $d_{C o m}=2 \times d_{A C}$.

For each scenario 100 different simulations lasting 400 seconds each are run. The results are presented with $95 \%$ confidence intervals. In order to conduct our simulations, we used a value of max_slots $=4$. This value has been defined after multiple tests that revealed it was the best value for a compromise between high throughput and latency. In fact, increasing max_slots would improve the throughput of the system and lower the number of collisions since readers would have more available slots to chose from. But in return, the latency would be increased as well since an incremented number of slots would also be unoccupied, uselessly widening the length of the frame. After numerous tests a value of max_slots $=4$ was chosen. The values of $T_{\text {beacon }}=5 \mathrm{~ms}$ and $T_{C R T}=460 \mathrm{~ms}$ are also defined according to [9], [5], [4], [8], [16]. Performance metrics for RFID system algorithms used for the characterization of our proposal are clearly introduced in [17].

\section{A. Collisions}

We distinguish two types of collisions: channel access collision and reading collisions. Channel access collision are observed in the case where multiple readers chose the same beaconing slot or when different readers in the same vicinity fail to chose different slots and channels. Reading collisions are detected when multiple readers access the tags in their intersecting surroundings at the same time. When the former occurs we consider an unsuccessful query section happened and tags were not read. The latter ones are not considered since according to our work and the one used to compare they cannot happen since readers in that situation are disabled by the respective algorithms.

Fig. 4a shows the number of collisions according to the density of readers deployed. Here we can witness that GDRA records considerably more collisions than DEFAR algorithm. This can be explained by the creation of an independent set setup process in DEFAR, during which after the case of a collision we maintain at least one reader enabled. Also within the span of a frame we have max_slots different reading sequences in DEFAR against a single one in GDRA. For a token [chosen_slot,chosen_channel], we may only have one single reader for a whole frame in its surrounding in GDRA since all readers on the same channel, regardless of their slots, get disabled. While in DEFAR, we can have as many readers as there are available slots in the design, thus cutting the number of collisions.

\section{B. Efficiency}

Efficiency defines the ratio of the number of successful query sections (SQS) over number of attempted query sections (AQS) as follows: $E f f=\frac{S Q S}{A Q S}$. Such a metric assesses how well protocols avoid collisions.

Fig. 4b demonstrates the efficiency of DEFAR and GDRA. Previous results shown in Fig. 4a explain the shapes of the efficiency plots. Since DEFAR has less collisions than GDRA, it induces a better efficiency with DEFAR outperforming GDRA. The smaller number of collisions result in a better efficiency for DEFAR making it a more suitable protocol for dense environments.

\section{Jain's fairness index}

Jain's fairness index [18] allows the evaluation on how fairly the throughput is distributed among the different readers in the system. It is computed as : $I_{J a i n}=\frac{\left|\sum_{i=1}^{n} x_{i}\right|^{2}}{n \times \sum_{i=1}^{n} x_{i}^{2}}$ where $x_{i}$ is the throughput of the $\mathrm{i}$-th reader and $n$ is the number of readers. When the throughput is equal for all 




(a) Collisions (lower is better)

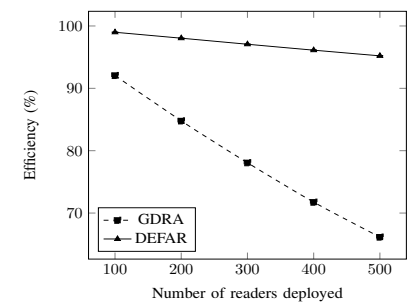

(b) Efficiency (higher is better)

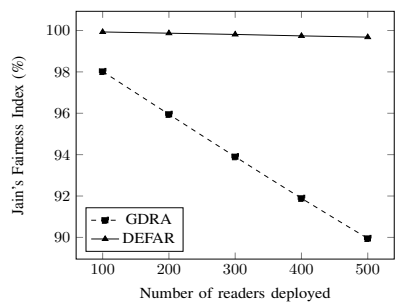

(c) Jain's Fairness (higher is better)

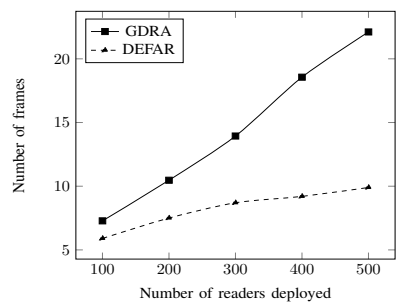

(d) Coverage delay (lower is better)

Figure 4: Performance evaluation results

readers, $I_{\text {Jain }}=1$ and in the worst case $I_{\text {Jain }}=\frac{1}{n}$.

Fig.4c displays the Jain's Fairness Index. We can first witness that both protocols have a really high fairness index never dropping under $90 \%$. But DEFAR is fairer since it favors previously failed readers to access the channel, balancing the queries between concurring readers.

\section{Coverage delay}

Coverage delay presents the minimal time needed to read, at least once, all tags that are in reading range of the readers in the system. The smaller the value of coverage delay, the faster and more efficient the protocol is.

Fig. 4d shows the evolution of the number of read tags throughout the frames. In the case of GDRA, we can see that it can take up to 23 frames in order to successfully read all covered tags. While in the case of DEFAR, all covered tags can be read within less than 10 frames. The higher efficiency as well as the better collisions management and fairness of DEFAR makes it more versatile for dense environments.

\section{DISCUSSIONS}

\section{A. Propagation model}

Throughout the modeling of our algorithm and the extensive testing conducted, we used an isotropic propagation model. Such a model is usually considered for simplicity purposes since the proposals are regarding collisions detection mechanisms. As seen in Figures 1a, 1b and 1c, 1d the reading and interference ranges of readers are represented as concentric circles around the considered reader.

In future works, we plan to extend our analysis using more realistic propagation models. First, the dedicated communication channel used for reader-to-reader coordination has to take into account the deployment environment. In urban or industrial settings, there might be readers that are not neighboring on this channel but generating reading collisions. Similarly, different types of RFID beam antennas are found in practical systems, which can modify the patterns of competing readers. We believe that DEFAR will still be the most efficient and fair approach, though collisions have to be evaluated under these models. The trade-off between reading efficiency and energy consumption is also interesting to investigate, in particular if the communication power on the coordination channel is a parameter.

\section{B. Mobility}

In order to explore the behavior of our proposed algorithm in different environments, we simulated different configurations considering static/moving readers and/or tags. This allowed us to witness the incidence of mobility on our proposal regarding main metrics such as collisions and fairness. For general purposes we considered a random waypoint mobility model, in future works more precise mobility models could be tested to check their incidence. Simulations were run with the same parameters as in Section V.

Fig. 5a displays the number of collisions in the system according to the number of readers deployed using different mobility environments. The number of collisions increases with the number of deployed readers as well. The number of colliding readers is not affected by the mobility factor, similar results are obtained with the different settings.

Fig. $5 \mathrm{~b}$ represents the efficiency of the proposal over the settings tested. Regarding the previous results on collisions we can explain the similar results obtained with efficiency observed here. Despite the shape of the curves, the efficiency still remains high with a lowpoint of $95.21 \%$ with the most dense deployments.

Fig. 5c shows the Jain's Fairness Index of our proposal with different mobility schemes considered. We observe that the fairness slowly decreases with a raising number of readers deployed but still remains with performances close to $100 \%$. It is interesting to see that we have two different shapes here, one for the case where the readers are moving and another where the readers are static. In the case of static readers, the performances are slowly diminishing whereas in the case of mobile readers the drop is more fair. This means that our proposal actually shows better performances regarding the fairness in a mobile scheme.

Simulation results show that DEFAR is not really affected by the mobility factor. It even performs better in terms of fairness in a mobile environment. Collisions performances are not altered and remain very satisfying. Such results prove that our proposal is relevant in uses cases that require the deployment of a dense and mobile RFID system as the ones presented in the introduction regarding the monitoring of warehouses using mobile readers hand-held or mounted on autonomous robots. 


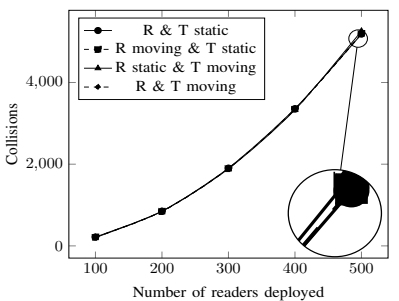

(a) Collisions

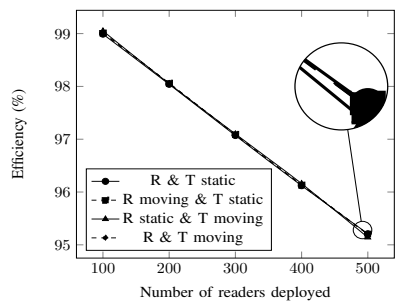

(b) Efficiency



(c) Jain's Fairness Index

Figure 5: Mobility performances evaluation

\section{CONCLUSION}

This paper presents DEFAR RFID anticollision protocol, a TDMA-based distributed multichannel protocol intended to reduce the collisions in RFID systems while maintaining a high level of fairness and efficiency. DEFAR leverages on the possible use of multiple frequencies to access the tags and on the ability to neighboring readers to communicate together. DEFAR is compliant with ETSI EN 302 208 [9] standards, allowing it to take advantage of the multichannel characteristic and being versatile for its use in different environments. As shown, DEFAR outperforms state of the art protocols. DEFAR manages the medium access by ensuring that in case of a collision at least one reader accesses the channel thus reducing the number of colliding readers and improving coverage speed. Also thanks to the definition of different priority levels, DEFAR improves the fairness among readers. Extensive simulations highlighted the compelling advantages of DEFAR over GDRA which is presented as the best anticollision protocol for RFID.

Other simulations made with mobile readers and/or tags also proved the high level of performance in these different environments. These simulations also show that the performance metrics levels are very similar whether in a mobile or static environment.

The next steps of this work will enhance DEFAR to leverage on the specificity of RFID collisions and also investigate the energy consumption of DEFAR protocol with different RFID materials in order to setup an autonomous energy harvesting RFID system. Experiments over a real RFID testbed are also considered in order to evaluate the physical layer interference and variations on our proposed algorithm. Finally, we will focus on the integration of RFID systems in a larger multihop wireless network.

\section{REFERENCES}

[1] K. Finkenzeller, RFID Handbook: Fundamentals and Applications in Contactless Smart Cards and Identification. John Wiley \& Sons, 2003, ch. 3, pp. 29-61.

[2] D. Engels and S. Sarma, "The reader collision problem," in IEEE Int. Conf. on Systems, Man and Cybernetics, 2002.

[3] E. S. specification, "EPC TM radio-frequency identity protocols class-1 generation-2 UHF RFID protocol for communications at $860 \mathrm{mhz}-960 \mathrm{mhz}$ version 1.2.0," 2007.
[4] M. V. Bueno-Delgado, R. Ferrero, F. Gandino, P. PavonMarino, and M. Rebaudengo, "A geometric distribution reader anti-collision protocol for rfid dense reader environments," IEEE Trans. on Automation Science and Engineering, 2013.

[5] R. Ferrero, F. Gandino, B. Montrucchio, and M. Rebaudengo, "Fair anti-collision protocol in dense rfid networks," in Int. EURASIP Workshop on RFID Technology, 2010.

[6] E. Hamouda, N. Mitton, and D. Simplot-Ryl, "Reader anticollision in dense rfid networks with mobile tags," in Int. Conf. on RFID-Technologies and Appli. RFID-TA, 2011.

[7] S. Birari and S. Iyer, "Pulse : A mac protocol for rfid networks," in Proc. of Int. workshop on RFID and Ubiquitous Sensor Networks, USN, 2005.

[8] I. Amadou and N. Mitton, "Hamac: High adaptive mac protocol for denserfid reader-to-reader networks," in Int. Conf. on Ad Hoc Networks, AdHocNets, 2015.

[9] ETSI, "Electromagnetic compatibility and radio spectrum matters erm radio frequency identification equipment operating in the band $865 \mathrm{mhz}$ to $868 \mathrm{mhz}$ with power levels up to 2 w part 2: Harmonized en covering the essential requirements of article 3.2 of the $r$ and tte directive," 2011.

[10] G. Khandelwal, K. Lee, A. Yener, and S. Serbetli, "Asap: a mac protocol for dense and time-constrained rfid systems," EURASIP journal on Wireless Comm. and Networking, 2007.

[11] J. Myung, W. Lee, and J. Srivastava, "Adaptive binary splitting for efficient rfid tag anti-collision," IEEE Com. Letters, 2006.

[12] J.-B. Eom, T.-J. Lee, R. Rietman, and A. Yener, "An efficient framed-slotted aloha algorithm with pilot frame and binary selection for anti-collision of rfid tags," IEEE Com. Letters, 2008.

[13] J. Waldrop, D. W. Engles, and S. E. Sarma, "Colorwave: An anticollision algorithm for the reader collision problem," in Proc. of IEEE Int. Conf. on Com., ICC, 2003.

[14] J. Waldrop, D. W. Engels, and S. E. Sarma, "Colorwave: an anticollision algorithm for the reader collision problem," in IEEE Int. Conf. on Com., ICC., 2003.

[15] K. Jamieson, H. Balakrishnan, and Y. Tay, "Sift: A mac protocol for event-driven wireless sensor networks," in Wireless Sensor Networks. Springer, 2006.

[16] I. Amadou, A. A. Mbacké, and N. Mitton, "How to improve csma-based mac protocol for dense rfid reader-to-reader networks?" in ADHOC-NOW, 2014.

[17] F. Gandino, R. Ferrero, B. Montrucchio, and M. Rebaudengo, "Evaluation criteria for reader-to-reader anti-collision protocols," Politecnico di Torino, Tech. Rep., 2012.

[18] R. Ferrero, F. Gandino, B. Montrucchio, and M. Rebaudengo, "A fair and high throughput reader-to-reader anticollision protocol in dense rfid networks," IEEE Trans. on Industrial Informatics, 2012. 